Linköping Studies in Science and Technology.

Thesis No. 1743

\title{
Group classification of linear Schrödinger equations by the algebraic method
}

\author{
Célestin Kurujyibwami
}

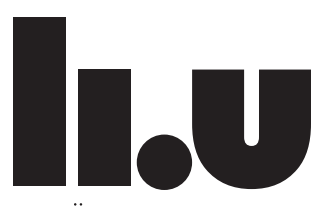

LINKÖPING UNIVERSITY

Department of Mathematics

Division of Mathematics and Applied Mathematics

Linköping University, SE-581 83 Linköping, Sweden

Linköping 2016 
Linköping Studies in Science and Technology.

Thesis No. 1743

\section{Group classification of linear Schrödinger equations}

by the algebraic method

Célestin Kurujyibwami

celestin.kurujyibwami@liu.se

www.mai.liu.se

Mathematics and Applied Mathematics

Department of Mathematics

Linköping University

SE-581 83 Linköping

Sweden

ISBN 978-91-7685-810-3ＩSSN 0280-7971

Copyright ( 2016 Célestin Kurujyibwami

Printed by LiU-Tryck, Linköping, Sweden 2016 


\section{Abstract}

This thesis is devoted to the group classification of linear Schrödinger equations. The study of Lie symmetries of such equations was initiated more than 40 years ago using the classical Lie infinitesimal method for specific types of real-valued potentials. In first papers on this subject, most attention was paid to dynamical transformations, which necessarily change the time and space variables. This is why phase translations were missed. Later, the study of Lie symmetries was extended to nonlinear Schrödinger equations. At the same time, the group classification problem for the class of linear Schrödinger equations with complex potentials remains unsolved.

The aim of the present thesis is to carry out the group classification for the class of linear Schrödinger equations with complex potentials. These potentials are nowadays important in quantum mechanics, scattering theory, condensed matter physics, quantum field theory, optics, electromagnetics and so forth. We exhaustively solve the group classification problem for space dimensions one and two.

The thesis comprises two parts. The first part is a brief review of Lie symmetries and group classification of differential equations. Next, we outline the equivalence transformations in a class of differential equations, normalization properties of such class and the algebraic method for group classification of differential equations.

The second part consists of two research papers. In the first paper, the algebraic method is applied to solve the group classification problem for $(1+1)$-dimensional linear Schrödinger equations with complex potentials. With this technique, the problem of the group classification of the class under study is reduced to the classification of certain subalgebras of its equivalence algebra. As a result, we find that the inequivalent cases are exhausted by eight families of potentials and we give the corresponding maximal Lie invariance algebras.

In the second paper we carry out the preliminary symmetry analysis of the class of linear Schrödinger equations with complex potentials in the multi-dimensional case. Using the direct method, we find the equivalence groupoid and the equivalence group of this class. Due to the multi-dimensionality, the results of the computations are quite different from the ones presented in Paper I. We obtain the complete group classification of $(1+2)$-dimensional linear Schrödinger equations with complex potentials. 



\section{Populärvetenskaplig sammanfattning}

Avhandlingen behandlar gruppklassificeringen av linjära Schrödingerekvationer. Studiet av sådana ekvationers Lie symmetrier påbörjades för mer än 40 år sedan. Man använde då Lies klassiska infinitesimala metod och begränsade sig till speciella typer av reellvärd potential. De första arbetena ägnades huvudsakligen åt dynamiska transformationer. Eftersom dessa nödvändigtvis ändrar både tidsoch rumsvariablerna behandlades inte fastranslationer. Senare utvidgades studierna till icke-linjära Schrödingerekvationer, men gruppklassificeringsproblemet för klassen av linjära Schrödingerekvationer med komplex potential förblev olöst.

Avhandlingens syfte är att undersöka gruppklassificeringen för klassen av linjära Schrödingerekvationer med komplex potential. Dessa potentialer är viktiga för tillämpningarna inom t ex kvantmekanik, spridningsteori, fysik för kondenserade material, kvantfältteori, optik och elektromagnetism. Vi löser gruppklassificeringproblemet fullständigt för rumsdimensionerna ett och två.

Avhandlingen har två delar. Den första delen är en kortfattad översikt över Liesymmetrier och gruppklassificering av differentialekvationer. Vidare skisserar vi ekvivalenstransformationerna i en klass av differentialekvationer, normaliseringsegenskaperna hos klassen och den algebraiska metoden för gruppklassificering av differentialekvationer.

Den andra delen består av två artiklar. I den första artikeln används den algebraiska metoden för att lösa gruppklassificeringproblemet för klassen av (1+1)dimensionella linjära Schrödingerekvationer med komplex potential. Med den tekniken reduceras detta problem till problemet att klassificera vissa delalgebror av klassens ekvivalensalgebra. Som resultat finner vi att de icke-ekvivalenta fallen uttöms av åtta familjer av potentialer och vi ger de motsvarande maximala Lie invariansalgebrorna.

I den andra artikeln gör vi den preliminära symmetrianalysen av klassen av linjära Schrödingerekvationer med komplex potential i det flerdimensionella fallet. Genom att använda den direkta metoden finner vi klassens ekvivalensgruppoid och ekvivalensgrupp. På grund av flerdimensionaliteten är resultaten av beräkningarna mycket olika dem, som presenteras i den första artikeln. Vi finner den fullständiga gruppklassificeringen av (1+2)-dimensionella linjära Schrödingerekvationer med komplex potential. 



\section{Acknowledgments}

I would like to express my deapest gratitude to my supervisors, Peter BasarabHorwath and Professor Roman Popovych for their invaluable guidance and advice throughout the writing of this thesis. They introduced me to the subject and have been providing me the required support along the way.

I thank Bengt-Ove Turesson, Björn Textorius, Meaza, Theresa and all the administrative staff of the Department of Mathematics for their help whenever needed.

I express my gratitude to the International Science programme (ISP) for their financial support through the East African Universities Mathematics programme (EAUMP) network. In particular, I would like to thank Leif Abrahamsson, Pravina Gajjar, Karangwa Desiré and Michael Gahirima.

My thanks go to my fellow PhD students for making Linköping such a nice place to live and work.

I want also to thank the Goverment of Rwanda and the University of Rwanda for giving me the opportunity to pursue further studies and providing me study leave.

Finally, I am grateful to my family for their support. Thank you my wife Dancille and my children Ineza and Hirwa for accepting the pain of missing me during the studies.

Linköping, February, 2016

Célestin Kurujyibwami 



\section{Contents}

1 General introduction 1

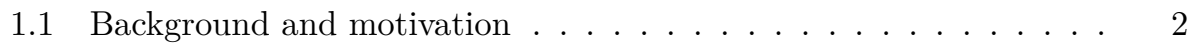

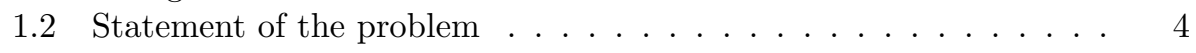

1.3 Thesis outline ....................... 4

I Theoretical background $\quad 7$

2 Group classification problem 9

2.1 Jet bundles and differential equations . . . . . . . . . . . . . . 10

2.1.1 Symmetry vector fields and their prolongations . . . . . . . 12

2.1.2 Nondegenerate systems of differential equations . . . . . . . 14

2.1.3 Classes of differential equation . . . . . . . . . . . 15

2.2 Equivalence transformations and normalization properties . . . . . 16

2.2.1 Equivalence transformations . . . . . . . . . . . 16

2.2.2 Normalization properties of classes of differential equations 18

2.3 Summary of the papers . . . . . . . . . . . . . . . . 19

$\begin{array}{ll}\text { Bibliography } & 21\end{array}$

II Research papers $\quad 23$

I Group classification of (1+1)-dimensional linear Schrödinger equations $\quad \mathbf{2 5}$

1 Introduction . . . . . . . . . . . . . . . 26 
2 Group classification in classes of differential equations . . . . . . . 29

3 Uniformly semi-normalized classes . . . . . . . . . . . . . . 32

4 Equivalence groupoid . . . . . . . . . . . . . . . . . . . 37

5 Analysis of determining equations for Lie symmetries . . . . . . . . 41

6 Group classification . . . . . . . . . . . . . . . . . . . . . 44

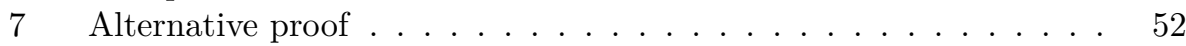

8 Subclass with real-valued potentials . . . . . . . . . . . 56

9 Conclusion . . . . . . . . . . . . . . . . 58

$\begin{array}{ll}\text { References } & 61\end{array}$

II Group classification of multidimensional linear Schrödinger equa$\begin{array}{ll}\text { tions } & 65\end{array}$

1 Introduction . . . . . . . . . . . . . . . . 66

2 Equivalence groupoid . . . . . . . . . . . . . . . . . . 69

3 Equivalence group and equivalence algebra . . . . . . . . . . . . . 73

4 Determining equations for Lie symmetries . . . . . . . . . . . . 74

$5 \quad$ Further properties of Lie symmetry algebras . . . . . . . . . . . . . 77

6 Group classification of $(1+2)$-dimensional linear Schrödinger equations ....................... 79

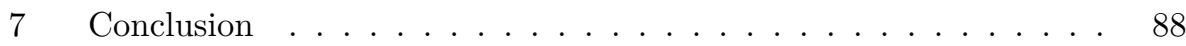

$\begin{array}{ll}\text { References } & 91\end{array}$ 


\section{1}

\section{General introduction}

Many physical phenomena are modelled using systems of differential equations in which certain parameters appear. These parameters may be constants or they may be functions: they are called arbitrary elements of the systems. As an example we have the nonlinear wave equation in $(1+1)$-space-time

$$
u_{t t}-u_{x x}=F\left(t, x, u_{x}\right)
$$

where $F\left(t, x, u_{x}\right)$ is an arbitrary smooth function of its arguments. The arbitrary element in this equation is the function $F\left(t, x, u_{x}\right)$.

In our analysis, the presence of arbitrary elements in the differential equation(s) leads to the requirement of an auxiliary system of differential equations which specify certain properties of these arbitrary elements. In the example of the nonlinear wave equation above, we note that the arbitrary element $F\left(t, x, u_{x}\right)$ is a function of $t, x, u_{x}$ but is independent of the function $u$ and the derivatives $u_{t}, u_{t x}$. Thus we can add to the above equation the auxiliary system

$$
\frac{\partial F}{\partial u}=0, \quad \frac{\partial F}{\partial u_{t}}=0, \quad \frac{\partial F}{\partial u_{t x}}=0
$$

and our "full system", for our example is then

$$
u_{t t}-u_{x x}=F\left(t, x, u_{x}\right), \quad F_{u}=F_{u_{t}}=F_{u_{t x}}=0 .
$$

Our analysis of such systems with arbitrary elements together with the auxiliary system therefore treats the equations as classes of equations rather than as a single system with a fixed choice of nonlinearities.

The idea of analysing of differential equations according to their symmetry properties is due to Sophus Lie [14], who created the theory of symmetries of differential equations. One of the deep results of his theory is an algorithmic method 
for finding (point) symmetries of differential equations. The basic questions in the Lie approach are: (1) what symmetries does a given equation possess? (2) given a group (in a given representation), what differential equations of a given order and type admit this group as a group of symmetries?

The analysis of equations using symmetries is in general a very difficult problem to solve, particularly if one attempts to apply Lie's symmetry algorithm. In simple cases, where the arbitrary elements depend on one variable only, but not on any derivative, direct application of Lie's method can be successful. However, if the arbitrary elements contain derivatives, then this direct approach is extremely difficult or even doomed to failure. It is in such cases that one can apply what we call the algebraic approach. This approach involves the classification of low-dimensional subalgebras of the equivalence algebra corresponding to the equivalence group of the class under study (these terms will be made more precise in the dissertation) and it is a synthesis of the infinitesimal Lie method (based on the invariance criterion), the technique of equivalence transformations and the analysis of classification of low-dimensional subalgebras.

In this thesis we carry out a group classification of the class of linear Schrödinger equations with a potential (which is the arbitrary element for this class, and which may be real or complex) given by

$$
i \psi_{t}+\psi_{a a}+V(t, x) \psi=0
$$

and which we refer to shortly as $\operatorname{LinSchEq}_{V}$. Here $t$ and $x=\left(x_{1}, x_{2}, \ldots, x_{n}\right)$ are the real independent variables, $\psi$ is a complex-valued function (referred to as the dependent variable) and $V$ is an arbitrary smooth real- or complex-valued potential depending on $t$ and $x$. Throughout this thesis the subscripts $t$ and $a$ denote differentiation with respect to $t$ and $x_{a}$, where the indices $a, b, c$, and $d$ run from 1 to $n$, and we use summation convention over repeated indices.

\subsection{Background and motivation}

The group classification of differential equations dates back to Sophus Lie's group classification of differential equations (Lie actually used what we now call Lie algebras of vector fields). In the 1870's Lie began an investigation of continuous group of transformations leaving differential equations invariant (actually mapping solutions of an equation to solutions of the same equation) and created what is known today as symmetry analysis of differential equations. It is within this theory that solutions of differential equations were derived in a completely algorithmic way and Lie gave a complete symmetry classification of scalar ordinary differential equations and possible symmetry reductions [14]. Despite these successes, Lie's approach to differential equations was not properly exploited until 1909 when Bateman showed that the free wave equation was invariant under the conformal group. However, it was only some fifty years later that Lie's approach was revived and began to be exploited systematically by L.V.Ovsiannikov [22] and other authors, who started to analyze systematically the methods of symmetry analysis of differential equations and to construct exact solutions. From the early 
1960 's onwards Lie symmetry analysis of differential equations became an area of intensive research producing many new results (although quite a few of these had in fact been discovered by Lie). Now there are many textbooks and research papers on the subject (see for instance $[3,21,4,9,20]$ ) and the Lie approach has become quite a standard method.

The study of linear Schrödinger equations from the symmetry point of view started in the 1970s. The usual Lie symmetry group of the $(1+3)$-dimensional free linear Schrodinger equation (i.e. $V=0$ ) was computed by Niederer in 1972 using the Lie infinitesimal method [17]. Niederer showed that, after factoring out the trivial part of the symmetry algebra, the Lie group of point transformations admitted by this equation is a 14-parameter locally Lie group generated by the Galilei group, the dilation and a group of projective transformations. In 1973 he calculated the maximal kinematical invariance group of the harmonic oscillator. At the same time he performed the analysis of the class $\operatorname{LinSchEq}_{V}$ with linear potential and he showed that the invariance groups for both the harmonic and linear potential are isomorphic to the free particle invariance group [18]. Then in 1974 he treated the group classification problem for the class $\operatorname{LinSchEq}_{V}$ for an arbitrary real-valued potential $V=V(t, x)$ in several dimensions $(n \leqslant 3)$ [19].

In [5] Boyer, using Lie's symmetry approach considered the group classification of the class LinSchEq $q_{V}$ with an arbitrary real-valued time-independent potential in three dimensions, $V=V\left(x_{i}\right)$. He found a non trivial symmetry algebra for an equation from the class $\operatorname{LinSchEq}_{V}$ of first order differential operator. He established there that the maximum dimension of a symmetry algebra is nine and that this maximum occurs only for the four families of potentials corresponding to the free particle, the harmonic oscillator, the repulsive oscillator and the case of free fall (linear operator), that is $V=0, V=k\left(x^{2}+y^{2}\right), V=-k\left(x^{2}+y^{2}\right)$ respectively where and $V=a x$, where $k \geqslant 0$ and $a \neq 0$. Furthermore, he showed that the algebras of maximal dimension are isomorphic, thus obtaining a generalization of Niederer's results.

In a series of works on the class $\operatorname{LinSchEq}_{V}$, Miller investigated symmetry properties and separation of variables for this class for both the case of $V=0$ and time dependent potentials [15].

With the further development of symmetry methods in the group classification of differential equations, more sophisticated tools were developed for looking at the more involved problems of group classification. Among the improvements in the approaches were the technique of equivalence transformations and the classification of low-dimensional subalgebras.

The technique based on equivalence transformations was introduced by Ovsiannikov [22] and become a powerful tool, a tool on which our group classification relies. The main property of these equivalence transformations is that they transform an equation from a given class to another equation of the same class. They constitute a group called the equivalence group. Another notion which complements the equivalence transformations was introduced by Kingston and Sophocleous [11, 12] under the name form preserving transformations, which are referred to as admissible transformations in [24]. The set of admissible transformations forms a groupoid and we refer to it as the equivalence groupoid and its computation is based on the 
direct method. It is this equivalence groupoid that the second approach of the group classification of low dimensional subalgebras is based on.

The relation between the equivalence groupoid and the equivalence group for a given class influences the choice of the appropriate technique for group classification and this leads to an efficient way of presenting the results $[24,13]$. It simplifies the process of classifying non trivial Lie symmetries within this class. More details on the use of the above approaches can be found in $[3,10,8,7]$, where the problem of the group classification of nonlinear Schrödinger equations is considered.

In this thesis we contend that there is a need to look at the group classification of the class $\operatorname{LinSchEq}_{V}$ using the equivalence group rather than just Lie's method. This is because the use of Lie's method does not guarantee the complete group classification of the class under study since it misses discrete equivalence transformations. Moreover, complex potentials have been shown to be of interest in mathematical-physics, quantum physics, optics, nuclear physics, as can be seen in $[2,1,6,16]$. Finally, a complete group classification of the class $\operatorname{LinSchEq}_{V}$ from our point of view is desirable in order to give a unified presentation of results.

\subsection{Statement of the problem}

Let $\operatorname{LinSchEq}_{V}$ be the class of linear Schrödinger equations with complex potentials of the form

$$
i \psi_{t}+\psi_{a a}+V(t, x) \psi=0,
$$

where the subscripts $t$ and $a$ denote differentiation with respect to $t$ and $x_{a}, t$ and $x=\left(x_{1}, x_{2}, \ldots, x_{n}\right)$ are the real independent variables, $\psi$ is the complex dependent variable and $V$ is an arbitrary smooth complex-valued potential depending on $t$ and $x$, the indices $a, b, c$, and $d$ run from 1 to $n$, and we use summation convention over repeated indices. We solve the complete group classification problem of the class $\operatorname{LinSchEq}_{V}$. In order to do this we first find the equivalence groupoid and the equivalence group of the class $\operatorname{LinSchEq}_{V}$. We then determine the kernel invariance algebra of the class $\operatorname{LinSchEq}_{V}$, that is the Lie algebra (of vector fields) which is admitted as a symmetry algebra for all choices of potential $V$. Finally we describe all inequivalent potentials admitting Lie symmetry extensions of the kernel invariance algebra. Exploiting the relation between the equivalence groupoid and the equivalence group of the class $\operatorname{LinSchEq}_{V}$ we reduce the group classification problem of the class $\operatorname{LinSchEq}_{V}$ to an analysis of low-dimensional subalgebras of equivalence transformations (generated by one parameter groups of equivalence transformations) that are projectable on the space of variables.

\subsection{Thesis outline}

This thesis comprises two parts: Chapter 2 and two research papers. In Chapter 2 we review some of the basic mathematical background of symmetry analysis and give the definitions of equivalence transformations, normalized classes and their properties. 
The two research papers are:

Paper I: Algebraic method for group classification of $(1+1)$-dimensional linear Schrödinger equations

This paper provides a new look at the group classification problem based on the algebraic method. We find the equivalence groupoid of the class of linear Schrödinger equations in $(1+1)$-space-time and show that this class is uniformly semi-normalized. Using the algebraic method we reduce the group classification to the classification of low-dimensional subalgebras of the corresponding equivalence algebra. A complete list of inequivalent potentials that admit Lie symmetry extensions is constituted by eight families of potentials.

\section{Paper II: Group classification of multidimensional linear Schrödinger equations with algebraic method}

In this paper, we study the group classification of multidimensional linear Schrödinger equations with complex potentials within the framework of the algebraic approach. Using the direct method, we derive the equivalence groupoid of the class of these equations, which possesses the uniformly semi-normalization property. This influences the applicability of the algebraic method and reduces the group classification of the equations to the classification of appropriate subalgebras of the associated equivalence algebra. In order to demonstrate the convenience and the performance of the method, inequivalent Lie symmetry extensions together with the corresponding potentials are listed for the case of $(1+2)$-dimensions. 



\section{Part I}

Theoretical background 



\section{Group classification problem}

Group classification of differential equations means that one characterizes (or tries to characterize) differential equations according to the symmetry properties possessed by them. For a given equation, one can calculate the (infinitesimal) symmetries according to the algorithmic method given by Lie (see for instance [21]). One assumes a particular form for the vector fields that represent the symmetries and then one obtains a set of first-order partial differential equations. These defining equations are solved and the exact form of the symmetries are established. This then establishes the symmetry properties of that equation (at least as far as certain types of symmetries are concerned). However, great difficulties arise when one tries to characterize classes of differential equations, such as equations of the type

$$
u_{t}=F\left(t, x, u, u_{x}, u_{x x}\right) \text {, }
$$

where $F$ is allowed to be any (locally defined) smooth function of its arguments such that $F_{u_{x x}} \neq 0$. A direct "frontal attack" using just Lie symmetries leads to an untractable system of equations. The answer to this apparent impasse is to use equivalence transformations associated with the class. These are essentially transformations which transform the equations of a given class into another of the same class. They are used to give canonical forms for symmetries and inequivalent types of equations within a given class. Thus, for the Schrödinger equations

$$
i \psi_{t}+\psi_{a a}+V(t, x) \psi=0
$$

we will give a list of canonical representatives of the potentials $V(t, x)$ and the maximal symmetry algebras admitted by these different potentials. The maximal symmetry algebras are Lie algebras of vector fields which define symmetries of the 
Schrödinger equations and each algebra is maximal in the sense that there is no larger symmetry algebra containing it. We first start with some basic definitions and results which underpin our work.

\subsection{Jet bundles and differential equations}

Jet bundles are the natural setting for discussing symmetries of differential equations. A jet bundle over a manifold $M$ is essentially a space whose local coordinates are the local coordinates $\left(x^{1}, \ldots, x^{n}\right)$ of $M$ together with functions $\left(u^{1}, \ldots, u^{m}\right)$ on $M$ as well as their derivatives. Thus if the manifold is $\mathbb{R}^{2}$ and we have one function $u: \mathbb{R}^{2} \rightarrow \mathbb{R}$, then the jet bundle of order two, denoted by $J^{2}\left(\mathbb{R}^{2}, \mathbb{R}\right)$, is the space with local coordinates $\left(x^{1}, x^{2}, u, u_{1}, u_{2}, u_{11}, u_{12}, u_{22}\right)$, where $u_{1}, u_{2}$ denote the first-order derivatives $\partial_{1} u, \partial_{2} u$ with $\partial_{1}, \partial_{2}$ being the partial derivatives with respect to $x^{1}, x^{2}$ respectively, and $u_{11}, u_{12}, u_{22}$ denote the three second-order partial derivatives of $u$ : $u_{11}=\partial_{1}^{2} u, u_{12}=\partial_{12} u, u_{22}=\partial_{2}^{2} u$. The jet bundle $J^{2}\left(\mathbb{R}^{2}, \mathbb{R}\right)$ is the natural space on which to treat second-order partial differential equations for real-valued functions of two real variables. A rigorous definition of jet bundles and their properties can be found in the book by Saunders [25], [23]. Our exposition below is informal and follows that of Olver in [21].

For a smooth real-valued function $u\left(x^{1}, \ldots, x^{n}\right)$ of $n$ independent variables we have the $k-$ th order derivatives

$$
\partial_{J} u=\frac{\partial^{k} u}{\partial x^{j_{1}} \partial x^{j_{2}} \ldots \partial x^{j_{k}}}
$$

where $J$ stands for the unordered $k$-tuple of integers $J=\left(j_{1}, \ldots, j_{k}\right)$ and where the integers $j_{l}, l=1, \ldots, k$ are such that $1 \leqslant j_{l} \leqslant n . J$ is referred to as a multiindex and its length, denoted by $|J|$, is defined as $|J|=k$. The corresponding jet space is denoted by $J^{k}\left(\mathbb{R}^{n}, \mathbb{R}\right)$ and has local coordinates $\left(x^{1}, \ldots, x^{n}, u\right)$ together with all the partial derivatives $\partial_{J} u, 1 \leqslant|J| \leqslant k$. Thus, for instance, $J^{3}\left(\mathbb{R}^{2}, \mathbb{R}\right)$ has local coordinates $\left(x, y, u, u_{x}, u_{y}, u_{x x}, u_{x y}, u_{y y}, u_{x x x}, u_{x x y}, u_{x y y}, u_{y y y}\right)$. We define the 'zeroth' jet bundle $J^{0}\left(\mathbb{R}^{n}, \mathbb{R}\right)$ as being the space with local coordinates $(x, u)$.

If we now take $u: \mathbb{R}^{n} \rightarrow \mathbb{R}^{m}$ then, writing $u=\left(u^{1}, \ldots, u^{m}\right)$, the corresponding jet space of order $k$ is denoted by $J^{k}\left(\mathbb{R}^{n}, \mathbb{R}^{m}\right)$ and its local coordinates are $\left(x^{1}, \ldots, x^{n}, u^{1}, \ldots, u^{m}\right)$ together with all the partial derivatives up to order $k$ given by $\partial_{J} u^{i}$ for $1 \leqslant|J| \leqslant k$ and $1 \leqslant i \leqslant m$. The 'zeroth' jet bundle $J^{0}\left(\mathbb{R}^{n}, \mathbb{R}^{m}\right)$ is then the space with local coordinates $(x, u)$ with the 'zeroth' derivative of a function being just the function itself.

We refer to the space $\mathbb{R}^{n} \times \mathbb{R}^{m}$ with local coordinates $\left(x^{1}, \ldots, x^{n}, u^{1}, \ldots, u^{m}\right)$ as the underlying space. It is the graph space of the mapping $u: \mathbb{R}^{n} \rightarrow \mathbb{R}^{m}$. In the literature on symmetries of differential equations the $\left(x^{1}, \ldots, x^{n}\right)$ are called the independent variables and the $\left(u^{1}, \ldots, u^{m}\right)$ are called the dependent variables.

The jet bundles $J^{k}\left(\mathbb{R}^{n}, \mathbb{R}^{m}\right)$ can be given the structure of a differentiable manifold (see [25]) and they are also fibred manifolds: there is a map (the projection map) $\pi_{k}: J^{k}\left(\mathbb{R}^{n}, \mathbb{R}^{m}\right): \rightarrow \mathbb{R}^{n} \times \mathbb{R}^{m}$ which is surjective (it is a submersion) given 
by $\pi_{k}\left(x, u_{(k)}\right)=(x, u)$ where $(x, u)=\left(x^{1}, \ldots, x^{n}, u^{1}, \ldots, u^{m}\right)$ and $u_{(k)}$ stands for the collection of all partial derivatives of $u=\left(u^{1}, \ldots, u^{m}\right)$ up to and including order $k$, with the 'zeroth' derivatives being just the functions themselves. The fibre above $(x, u)$ is just the inverse image $\pi_{k}^{-1}(x, u)$ (see $\left.[25,23]\right)$. One also has the projections $\pi_{k, l}: J^{k}\left(\mathbb{R}^{n}, \mathbb{R}^{m}\right) \rightarrow J^{l}\left(\mathbb{R}^{n}, \mathbb{R}^{m}\right)$ for $k \geqslant l$ given by $\pi_{k, l}\left(x, u_{(k)}\right)=\left(x, u_{(l)}\right)$ and it is easy to verify that $\pi_{j, k} \circ \pi_{k, l}=\pi_{j, l}$ whenever $j \geqslant k \geqslant l$. We also have $\pi_{k, k}=\mathrm{id}$ and $\pi_{k}=\pi_{l} \circ \pi_{k, l}$ for all $k \geqslant l$.

The structure of a fibred manifold allows us to define diffeomorphisms of $J^{k}\left(\mathbb{R}^{n}, \mathbb{R}^{m}\right)$. Note that any diffeomorphism $\Phi: \mathbb{R}^{n} \times \mathbb{R}^{m} \rightarrow \mathbb{R}^{n} \times \mathbb{R}^{m}$ of the underlying space $\mathbb{R}^{n} \times \mathbb{R}^{m}$ induces a transformation of the derivatives of all orders of $u=\left(u^{1}, \ldots, u^{m}\right)$. The induced transformation on the jet space $J^{k}\left(\mathbb{R}^{n} \times \mathbb{R}^{m}\right)$ obtained in this way (by the usual chain rule) is called the $k$ th prolongation of $\Phi$. It is clear that the $k$-th prolongation of a diffeomorphism $\Phi$ will depend only on the local coordinates $\left(x, u_{(k)}\right)$. We use the notation $\left.\Phi\right|_{\left(x, u_{(k)}\right)}$ to denote the $k$-th prolongation of $\Phi$.

The jet bundles described above are endowed with vector fields $D_{i}, i=1, \ldots, n$ called the total derivatives and they are defined as follows: for the functions $P: J^{k}\left(\mathbb{R}^{n}, \mathbb{R}^{m}\right) \rightarrow \mathbb{R}$

$$
D_{i}=\frac{\partial P}{\partial x^{i}}+\sum_{\alpha=1}^{m} \frac{\partial P}{\partial u^{\alpha}}+\sum_{\alpha=1}^{m} \sum_{J} u_{J, i}^{\alpha} \frac{\partial P}{\partial u_{J}^{\alpha}},
$$

where the summation over $J$ is over all $k$-tuples $\left(j_{1}, \ldots, j_{k}\right)$ of all lengths $|J| \geqslant 1$. The sum is well defined since $P$ is a function of only a finite number of arguments. Here $u_{J, i}^{\alpha}$ is defined for $J=\left(j_{1}, \ldots, j_{k}\right)$ as

$$
u_{J, i}^{\alpha}=\frac{\partial u_{J}^{\alpha}}{\partial x^{i}}=\frac{\partial^{k+1} u^{\alpha}}{\partial x^{i} \partial x^{j_{1}} \ldots \partial x^{j_{k}}} .
$$

\section{Example 2.1}

The space on which second-order ordinary differential equations are defined is $J^{2}(\mathbb{R}, \mathbb{R})$. The underlying space is $\mathbb{R} \times \mathbb{R}$ with local coordinates $(x, y)$. The local coordinates on $J^{2}(\mathbb{R}, \mathbb{R})$ are then $\left(x, y, y^{\prime}, y^{\prime \prime}\right)$ and the total derivative with respect to $x$ is

$$
D_{x}=\frac{\partial}{\partial x}+y^{\prime} \frac{\partial}{\partial y}+y^{\prime \prime} \frac{\partial}{\partial y^{\prime}}+y^{\prime \prime \prime} \frac{\partial}{\partial y^{\prime \prime}} .
$$

\section{Example 2.2}

For $J^{2}\left(\mathbb{R}^{2}, \mathbb{R}\right)$ we have the underlying space $\mathbb{R}^{2} \times \mathbb{R}$ with local coordinates $(x, y, u)$ and the local coordinates on $J^{2}\left(\mathbb{R}^{2}, \mathbb{R}\right)$ are then $\left(x, y, u, u_{x}, u_{y}, u_{x x}, u_{x y}, u_{y y}\right)$. We 
have two total derivatives

$$
\begin{aligned}
& D_{x}=\frac{\partial}{\partial x}+u_{x} \frac{\partial}{\partial u}+u_{x x} \frac{\partial}{\partial u_{x}}+u_{x y} \frac{\partial}{\partial u_{y}}+u_{x x x} \frac{\partial}{\partial u_{x x}}+u_{x x y} \frac{\partial}{\partial u_{x y}}+u_{x y y} \frac{\partial}{\partial u_{y y}} \\
& D_{y}=\frac{\partial}{\partial y}+u_{y} \frac{\partial}{\partial u}+u_{x y} \frac{\partial}{\partial u_{x}}+u_{y y} \frac{\partial}{\partial u_{y}}+u_{y y y} \frac{\partial}{\partial u_{y y}}+u_{y y x} \frac{\partial}{\partial u_{x y}}+u_{x x y} \frac{\partial}{\partial u_{x x}} .
\end{aligned}
$$

Strictly speaking the total derivatives $D_{x}, D_{y}$ mentioned in these example are generalized vector fields rather than a vector field since the coefficient $y^{\prime \prime \prime}$ and $u_{x x x}, u_{x x y}, u_{y y y}$ appear. This problem can be circumvented by working on the infinite jet bundle $J^{\infty}\left(\mathbb{R}^{n}, \mathbb{R}^{m}\right)$ where the local coordinates include derivatives $u_{J}^{\alpha}$ of arbitrary order (that is with $|J|$ takes on all possible positive integer values). Functions on this infinite jet bundle are defined as being functions of only a finite number of non-zero arguments. A rigorous definition is given in [25], but we shall not need this construction. We do not use jet bundles other than in an informal way and the total derivatives are to be understood as generalized vector fields.

We think of systems of differential equations of order $k$ for $m$ unknown functions $\left(u^{1}, \ldots, u^{m}\right)$ in $n$ independent variables $\left(x^{1}, \ldots, x^{n}\right)$ as being given by smooth functions $L: J^{k}\left(\mathbb{R}^{n}, \mathbb{R}^{m}\right) \rightarrow \mathbb{R}^{p}$ where $k, m, n, p$ are positive integers. We have the following standard definition ( $[21])$

Definition 2.1. A system of $p$ differential equations of order $k$ for $m$ functions of $n$ independent variables is defined by a smooth function

$$
L\left(x, u, u_{(k)}\right)=0, \quad L: J^{k}\left(\mathbb{R}^{n}, \mathbb{R}^{m}\right) \rightarrow \mathbb{R}^{p}
$$

for some integer $p$. We say that the system is maximal rank if the Jacobian matrix of the function $L: J^{k}\left(\mathbb{R}^{n}, \mathbb{R}^{m}\right) \rightarrow \mathbb{R}^{p}$ has rank $p$ (this is Definition 2.30 in [21]).

\subsubsection{Symmetry vector fields and their prolongations}

In calculating symmetries of differential equations, we look at one-parameter symmetry groups which are generated (at least locally) by vector fields. The vector fields which are of interest here are those which arise from vector fields on the underlying manifold $\mathbb{R}^{n} \times \mathbb{R}^{m}$.

We define a symmetry of a system of partial differential equations as follows:

Definition 2.2. A symmetry of a system of differential equations of the form

$$
L\left(x, u, u_{(k)}\right)=0, \quad L: J^{k}\left(\mathbb{R}^{n}, \mathbb{R}^{m}\right) \rightarrow \mathbb{R}^{p}
$$

is a map $\Phi:(x, u) \rightarrow(\tilde{x}, \tilde{u})$ so that

$$
L\left(\tilde{x}, \tilde{u}, \tilde{u}_{(k)}\right)=0 \quad \text { whenever } \quad L\left(x, u, u_{(k)}\right)=0 .
$$

Definition 2.3. The map $\left(x, u_{(k)}\right) \rightarrow\left(\tilde{x}, \tilde{u}_{(k)}\right)$ induced by the map $\Phi:(x, u) \rightarrow$ $(\tilde{x}, \tilde{u})$ is called the $k$-th prolongation of $\Phi$, denoted by $\operatorname{pr}^{(k)} \Phi$. 
It is an essentially hopeless task to find symmetries of differential equations with this definition. However, using this definition we can restrict our attention to (locally defined) one-parameter groups generated by vector fields of the form

$$
X=\sum_{a=1}^{n} \xi^{a}(x, u) \partial_{x_{a}}+\sum_{\alpha=1}^{m} \eta^{\alpha}(x, u) \partial_{u^{\alpha}}
$$

The action of this vector field can be extended to an action on functions on the jet bundle $P: J^{k}\left(\mathbb{R}^{n}, \mathbb{R}^{m}\right) \rightarrow \mathbb{R}$. This extended action is called the $k$-th prolongation of the vector field $X$, and is denoted by $X_{(k)}$ and is defined by the formula

$$
X_{(k)}=\sum_{a=1}^{n} \xi^{a}(x, u) \partial_{x_{a}}+\eta^{\alpha}(x, u) \partial_{u^{\alpha}}+\sum_{\alpha=1}^{m} \sum_{J}^{m} \eta_{J}^{\alpha} \partial_{u_{J}^{\alpha}},
$$

The sum over $J$ is over all $k$-tuples $J$ with $|J| \geqslant 1$. The functions $\eta_{J}^{\alpha}$ are defined as follows for each $J=\left(j_{1}, j_{2}, \ldots, j_{k}\right)$ :

$$
\eta_{J}^{\alpha}=D_{J}\left(\eta^{\alpha}-\sum_{i=1}^{m} \xi^{i} u_{i}^{\alpha}\right)+\xi^{a} u_{J, a}^{\alpha}
$$

with $D_{J}=D_{j_{1}} D_{j_{2}} \ldots D_{j_{k}}$. Alternatively, they may be given recursively as

$$
\eta_{i}^{\alpha}=D_{i} \eta^{\alpha}-u_{a}^{\alpha} D_{i} \xi^{\alpha}, \quad \eta_{J, i}^{\alpha}=D_{i} \eta_{J}^{\alpha}-u_{J, a}^{\alpha} D_{i} \xi^{\alpha}
$$

for $|J| \geqslant 1$.

\section{Example 2.3}

For the vector field

$$
X=x \partial_{x}+2 u \partial_{u}
$$

defined on the underlying space $\mathbb{R} \times \mathbb{R}$ the first prolongation $X_{(1)}$ is

$$
X_{(1)}=x \partial_{x}+2 u \partial_{u}+\eta_{x} \partial_{u_{x}}=x \partial_{x}+2 u \partial_{u}+u_{x} \partial_{u_{x}}
$$

since $\eta_{x}=D_{x}\left(2 u-x u_{x}\right)+x u_{x x}$.

\section{Example 2.4}

The vector fields $X=\partial_{x}, Y=x \partial_{x}, Z=x^{2} \partial_{x}$ form the Lie algebra $\operatorname{sl}(2, \mathbb{R})$ and the commutation relations are $[Y, X]=-X,[Y, Z]=Z[X, Z]=2 Y$. Their second prolongations for the underlying space $\mathbb{R}^{2} \times \mathbb{R}$ are

$$
\begin{aligned}
& X_{(2)}=\partial_{x}, \quad Y_{(2)}=x \partial_{x}-u_{x} \partial_{u_{x}}-2 u_{x x} \partial_{u_{x x}}, \\
& Z_{(2)}=x^{2} \partial_{x}-2 u_{x} \partial_{u_{x}}-\left(2 u_{x}+4 x u_{x x}\right) \partial_{u_{x x}}
\end{aligned}
$$

For more details on these definitions and examples, we refer to [21]. 
Lemma 2.1. Let $\Phi_{X}(t)$ denote the local flow of a vector field $X$ on $\mathbb{R} \times \mathbb{R}^{m}$ and $\Phi_{X_{(k)}}(t)$ the local flow of $X_{(k)}$ on $J^{k}\left(\mathbb{R} \times \mathbb{R}^{m}\right)$, then

$$
\operatorname{pr}^{(k)} \Phi_{X}(t)=\Phi_{X_{(k)}}(t) .
$$

For a proof, see [21].

We can now define what we mean by an infinitesimal symmetry operator for a differential equation:

Definition 2.4. The vector field $X$ defined by

$$
X=\sum_{a=1}^{n} \xi^{a}(x, u) \partial_{x_{a}}+\sum_{\alpha=1}^{m} \eta^{\alpha}(x, u) \partial_{u^{\alpha}}
$$

is an infinitesimal point symmetry of a system of differential equations $L\left(x, u_{(k)}\right)=$ 0 , where $L: J^{k}\left(\mathbb{R}^{n}, \mathbb{R}^{m}\right) \rightarrow \mathbb{R}^{p}$, if the local diffeomorphism generated by the local flow $\Phi_{X}(t)$ of $X$ is a symmetry of this system.

The basic result on symmetries of differential equations is the following:

Theorem 2.1. If the (smooth) vector field $X$ defined on the underlying space $\mathbb{R}^{n} \times \mathbb{R}^{m}$ is an infinitesimal point symmetry of the system of differential equations $L\left(x, u_{(k)}\right)=0$, where $L: J^{k}\left(\mathbb{R}^{n}, \mathbb{R}^{m}\right) \rightarrow \mathbb{R}^{p}$ is of maximal rank, then

$$
X_{(k)} L=0 \quad \text { whenever } \quad L\left(x, u_{(k)}\right)=0 .
$$

If $X, Y$ are two infinitesimal point symmetries of the system of differential equations of maximal rank $L\left(x, u_{(k)}\right)=0$ then so is their commutator $[X, Y]$.

Here $X_{(k)} L=0$ means $X_{(k)} L_{q}=0$ for all $q=1, \ldots, p$.

Note that this result only says that if $\Phi_{X}(t)$ is a symmetry, then $X_{(k)} L=0$. It requires that, at least for $t \in I$ for some interval $I$ of zero, $\Phi_{X}(t) u$ to be a solution of $L\left(x, u_{(k)}\right)=0$ whenever $u$ is a solution. The converse statement requires the extra condition of nondegeneracy.

\subsubsection{Nondegenerate systems of differential equations}

Our work deals with non-degenerate systems of differential equations. We have the following definitions (see [21]):

Definition 2.5. A system of $k$-th order differential equations $L\left(x, u_{(k)}\right)=0$ is said to be locally solvable at the point $\left(x, u_{(k)}\right)$ for which $L\left(x, u_{(k)}\right)=0$ if there exists a smooth solution $u=f(x)$ of the system $L\left(x, u_{(k)}\right)=0$ which is defined in some neighbourhood of $x$ and satisfying $u_{(k)}=\operatorname{pr}^{(k)} f(x)$ where $\operatorname{pr}^{(k)} f(x)$ denotes the collection of all derivatives of $f(x)$ of order up to and including $k$.

The system is locally solvable if it is locally solvable at every point of the set $\left\{\left(x, u_{(k)}\right): L\left(x, u_{(k)}\right)=0\right\}$. 
Definition 2.6. A system of differential equations is said to be non-degenerate if it is locally solvable and is also of maximal rank at each point of the set $\left\{\left(x, u_{(k)}\right): L\left(x, u_{(k)}\right)=0\right\}$.

The importance of non-degeneracy is illustrated in the following theorem:

Theorem 2.2. Suppose $L\left(x, u_{(k)}\right)=0$ is a nondegenerate system of differential equations. A vector field $X$ on the underlying space $\mathbb{R}^{n} \times \mathbb{R}^{m}$ is an infinitesimal symmetry of the system if and only if

$$
X_{(k)} L=0 \quad \text { whenever } \quad L\left(x, u_{(k)}\right)=0 .
$$

For a proof, see [21].

\subsubsection{Classes of differential equation}

We now come to the concept of a class of equations. An evolution equation of second order is of the form

$$
u_{t}=F\left(t, x, u, u_{x}, u_{x x}\right)
$$

where $F$ is a smooth function of its arguments, and it belongs to a class of equations with the following conditions on $F$

$$
F_{u_{t}}=F_{u_{t x}}=0, \quad F_{u_{x x}} \neq 0 .
$$

We call $F$ an arbitrary element. We thus have a system of the form

$$
u_{t}=F\left(t, x, u, u_{x}, u_{x x}\right), \quad F_{u_{t}}=F_{u_{t x}}=0, \quad F_{u_{x x}} \neq 0 .
$$

This can be symbolized by

$$
L_{\theta}\left(t, x, u_{(2)}, \theta\left(t, x, u_{(2)}\right)\right)=0
$$

where $\theta=F$ and belongs to the set of (locally) smooth functions satisfying $\theta_{u_{t}}=$ $\theta_{u_{t x}}=0, \theta_{u_{x x}} \neq 0$. The functions $\theta$ are called arbitrary elements. Note that is a system of differential equations, there will in general be more than one arbitrary element.

In our work we follow [24], and we consider systems of differential equations

$$
\mathcal{L}_{\theta}: L\left(x, u_{(p)}, \theta_{(q)}\left(x, u_{(p)}\right)\right)=0,
$$

parameterized by the tuple of arbitrary elements $\theta\left(x, u_{(p)}\right)$, where $\theta$ denotes the tuple $\theta=\left(\theta^{1}, \ldots, \theta^{k}\right)$ and $\theta_{(q)}$ is the set of derivatives of $\theta$ up to and including order $q$. This tuple of arbitrary elements $\theta$ satisfies an auxiliary system of equations $\mathcal{S}$ which consists of equations of the form $S\left(x, u_{(p)}, \theta_{(q)}\left(x, u_{(p)}\right)\right)=0$. This auxiliary system may also be augmented by some non-vanishing condition written representatively as $\Sigma\left(x, u_{(p)}, \theta_{(q)}\left(x, u_{(p)}\right)\right) \neq 0$, which is taken to mean that no component of $\Sigma$ vanishes.

In this way, we have the class of systems of differential equations $\mathcal{L}_{\theta}$ where the arbitrary elements $\theta$ run through $\mathcal{S}$. We denote this class by $\left.\mathcal{L}\right|_{\mathcal{S}}$. 


\section{Example 2.5}

In the above example of an evolution equation we have $\theta=F$, the auxiliary system is $\mathcal{S}: \theta_{u_{t}}=0, \theta_{u_{t x}}=0$ and the non-vanishing condition is $\Sigma: \theta_{u_{x x}} \neq 0$.

\section{Example 2.6}

The class of nonlinear wave equations of the form

$$
u_{t t}=f\left(x, u_{x}\right) u_{x x}+g\left(x, u_{x}\right)
$$

is a single system with two arbitrary elements $\theta^{1}=f$ and $\theta^{2}=g$ depending on $x$ and $u_{x}$ respectively. The auxiliary system associated to this class is formed by the equations:

$$
\begin{aligned}
& \theta_{t}^{1}=\theta_{u}^{1}=\theta_{u_{t}}^{1}=\theta_{u_{t x}}^{1}=\theta_{u_{x x}}^{1}=0, \\
& \theta_{t}^{2}=\theta_{u}^{2}=\theta_{u_{t}}^{2}=\theta_{u_{t x}}^{2}=\theta_{u_{x x}}^{2}=0 .
\end{aligned}
$$

and the non-vanishing condition is $\Sigma: \theta^{1} \neq 0$.

\section{Example 2.7}

For the class $\operatorname{LinSchEq}_{V}$ of equations $i \psi_{t}+\psi_{a a}+V(t, x) \psi=0$, we have a single arbitrary element $\theta=V$ with the auxiliary system of the form

$$
\theta_{\psi}=\theta_{\psi^{*}}=\theta_{\psi_{x}}=\theta_{\psi_{x}^{*}}=\theta_{\psi_{t}}=\theta_{\psi_{t}^{*}}=\theta_{\psi_{t x}}=\theta_{\psi_{t x}^{*}}=\theta_{\psi_{t t}}=\theta_{\psi_{t t}^{*}}=0 .
$$

\subsection{Equivalence transformations and normalization properties}

Group classification theory of differential equations is mainly built upon the notion of equivalence transformations. In this section we look at equivalence transformations of classes of differential equations and some of their properties. More details can be found in $[3,13,24]$.

\subsubsection{Equivalence transformations}

We begin with admissible transformations ([24]):

Definition 2.7. If $\mathcal{L}_{\theta}$ and $\mathcal{L}_{\tilde{\theta}}$ are two systems belonging to the class $\left.\mathcal{L}\right|_{\mathcal{S}}$ we say an admissible transformation from $\mathcal{L}_{\theta}$ to $\mathcal{L}_{\tilde{\theta}}$ is an invertible point transformation 
of the underlying space having local coordinates $(x, u)$ which maps $\mathcal{L}_{\theta}$ to $\mathcal{L}_{\tilde{\theta}}$. We denote by $T(\theta, \tilde{\theta})$ the set of admissible transformations from $\mathcal{L}_{\theta}$ to $\mathcal{L}_{\tilde{\theta}}$.

The set of admissible transformations from $\mathcal{L}_{\theta}$ into the class $\left.\mathcal{L}\right|_{\mathcal{S}}$ is defined as the set $T\left(\theta,\left.\mathcal{L}\right|_{\mathcal{S}}\right)=\{(\tilde{\theta}, \varphi) \mid \tilde{\theta} \in \mathcal{S}, \varphi \in T(\theta, \tilde{\theta})\}$.

Definition 2.8. We define the set of admissible transformations in the class of differential equations as $\mathcal{G} \sim\left(\left.\mathcal{L}\right|_{\mathcal{S}}\right):=\{(\theta, \tilde{\theta}, \varphi) \mid \theta, \tilde{\theta} \in \mathcal{S}, \varphi \in \mathrm{T}(\theta, \tilde{\theta})\}$.

The set $\mathcal{G}^{\sim}$ satisfies the axioms of a groupoid and we call it the equivalence groupoid of the class $\left.\mathcal{L}\right|_{\mathcal{S}}$.

We note that in terms of the notation introduced here, the maximal local group of point symmetries of the system $\mathcal{L}_{\theta}$ for a fixed $\theta \in \mathcal{S}$ is just the set $G_{\theta}=T(\theta, \theta)$. We also have the kernel of the maximal point symmetry groups of systems for the class $\left.\mathcal{L}\right|_{\mathcal{S}}$ :

Definition 2.9. The kernel of the maximal point symmetry groups of systems for the class $\left.\mathcal{L}\right|_{\mathcal{S}}$ is given by

$$
G^{\cap}=\cap_{\theta \in \mathcal{S}} G_{\theta}
$$

This group lies at the heart of our approach: one first determines those symmetries that are admitted by every system $\mathcal{L}_{\theta}$ and then one looks at all admissible extensions.

We now come to another central object in our approach: the equivalence group. This is defined as follows (see [24]):

Definition 2.10. The (usual) equivalence group $G^{\sim}\left(\left.\mathcal{L}\right|_{\mathcal{S}}\right)$ of the class $\left.\mathcal{L}\right|_{\mathcal{S}}$ (of systems of differential equations over the underlying space $\mathbb{R}^{n} \times \mathbb{R}^{m}$ ) is defined as set of transformations $\Phi$ in the space $J^{p}\left(\mathbb{R}^{n}, \mathbb{R}^{m}\right) \times \mathcal{S}$ which are projectable onto the spaces $J^{l}\left(\mathbb{R}^{n}, \mathbb{R}^{m}\right)$ for $0 \leqslant l \leqslant p$. That is $\left.\Phi\right|_{\left(x, u_{(l)}\right)}$ is the $l$-th order prolongation of $\left.\Phi\right|_{(x, u)}$ for all $\theta \in \mathcal{S}$ for which $\Phi \theta \in \mathcal{S}$ and $\left.\Phi\right|_{(x, u)} \in T(\theta, \Phi \theta)$. Here $\left.\Phi\right|_{\left(x, u_{(l)}\right)}$ denotes the restriction of $\Phi$ to $J^{l}\left(\mathbb{R}^{n}, \mathbb{R}^{m}\right)$. We also denote this set by $G^{\sim}$.

We denote the projection operator by $\pi$, so that we have

$$
\pi: J^{p}\left(\mathbb{R}^{n}, \mathbb{R}^{m}\right) \times \mathcal{S} \rightarrow J^{p}\left(\mathbb{R}^{n}, \mathbb{R}^{m}\right) .
$$

Admissible transformations of the form $\left(\theta, \Phi \theta,\left.\Phi\right|_{(x, u)}\right)$ with $\theta \in \mathcal{S}$ and $\Phi \in G^{\sim}$ are said to be induced by the transformations of $G^{\sim}$.

We also have the projection

$$
\pi=\pi_{p} \circ \operatorname{proj}_{1}: J^{p}\left(\mathbb{R}^{n}, \mathbb{R}^{m}\right) \times \mathcal{S} \rightarrow J^{0}\left(\mathbb{R}^{n}, \mathbb{R}^{m}\right)=\mathbb{R}^{n} \times \mathbb{R}^{m} \text { for any } J^{p}\left(\mathbb{R}^{n}, \mathbb{R}^{m}\right) .
$$

This projects down to the underlying space.

Our method involves computing the equivalence groupoid $\mathcal{G}^{\sim}$ and the equivalence group $G^{\sim}$ directly (that is, in "finite form"). We also work with the equivalence algebra. This is defined as follows: 
Definition 2.11. The equivalence algebra of the class $\left.\mathcal{L}\right|_{\mathcal{S}}$, denoted by $\mathfrak{g}^{\sim}$ is the Lie algebra defined by all the (smooth) one-parameter subgroups of the equivalence group of $G^{\sim}$ of $\left.\mathcal{L}\right|_{\mathcal{S}}$.

The Lie algebra $\mathfrak{g}_{\theta}$ is the Lie algebra defined by all the (smooth) one-parameter subgroups of $G_{\theta}$. It is the maximal (in dimension) Lie invariance algebra of the system $\mathcal{L}_{\theta}$.

The kernel Lie algebra is the Lie algebra $\mathfrak{g}^{\cap}=\cap_{\theta \in \mathcal{S}} \mathfrak{g}_{\theta}$.

These definitions can be found in [24].

\subsubsection{Normalization properties of classes of differential equations}

The group classification problem for an arbitrary class $\left.\mathcal{L}\right|_{\mathcal{S}}$ is a complex and challenging undertaking. In general, one will only be able to achieve a partial classification. However, one can identify a criterion that leads to a complete classification.

Definition 2.12. The class $\left.\mathcal{L}\right|_{\mathcal{S}}$ is said to be normalized if its set of admissible transformations is induced by transformations from its (usual) equivalence group. That is, if for each triple $(\theta, \tilde{\theta}, \varphi) \in \mathcal{G}^{\sim}\left(\left.\mathcal{L}\right|_{\mathcal{S}}\right)$ there exists a transformation $\Phi \in G^{\sim}$ such that $\tilde{\theta}=\Phi \theta$ and $\varphi=\left.\Phi\right|_{(x, u)}$

It is said to be semi-normalized if $\mathcal{G}^{\sim}\left(\left.\mathcal{L}\right|_{\mathcal{S}}\right)$ is generated by the transformations from (usual) equivalence group $G^{\sim}$ of the whole class together with transformations from symmetry groups of initial or transformed systems. That is, if for each triple $(\theta, \tilde{\theta}, \varphi) \in \mathcal{G}^{\sim}\left(\left.\mathcal{L}\right|_{\mathcal{S}}\right)$ there exists a transformation $\Phi \in G^{\sim}$ and a transformation $\tilde{\varphi} \in G_{\theta}$ of the system $\mathcal{L}_{\theta}$ such that $\tilde{\theta}=\Phi \theta$ and $\varphi=\left.\Phi\right|_{(x, u)} \circ \tilde{\varphi}$. (see [13]).

Remark: It follows immediately from this definition that any normalized class is automatically semi-normalized.

The property of being semi-normalized leads to the following result:

Proposition 2.1. If $\left.\mathcal{L}\right|_{\mathcal{S}}$ is a semi-normalized class, then we have

$$
\operatorname{span} \bigcup_{\theta \in \mathcal{S}} \mathfrak{g}_{\theta} \subseteq \pi_{*} \mathfrak{g}^{\sim}
$$

Here $\pi_{*}$ denotes the tangent map of the projection map

$$
\pi=\pi_{p} \circ \operatorname{proj}_{1}: J^{p}\left(\mathbb{R}^{n}, \mathbb{R}^{m}\right) \times \mathcal{S} \rightarrow \mathbb{R}^{n} \times \mathbb{R}^{m} .
$$

Note that $\pi_{*}$ maps vector fields of the form

$$
Q=\xi^{a}(x, u) \partial_{x_{a}}+\eta^{\alpha}(x, u) \partial_{u^{\alpha}}+\zeta^{s}(x, u, \theta) \partial_{\theta^{s}}
$$

to vector fields of the form $\pi_{*} Q=\xi^{a} \partial_{x_{a}}+\eta^{\alpha} \partial_{u^{\alpha}}$ defined on the underlying space $\mathbb{R}^{n} \times \mathbb{R}^{m}$ with local coordinates $(x, u)$

It is this result that allows us to obtain a complete group classification. Without normalization property, we would have only a partial group classification. 
The method we employ in this thesis may be summed up as follows:

First, compute the equivalence groupoid and the equivalence group. Then establish semi-normalization for the class under investigation.

Secondly, calculate the equivalence algebra, the maximal Lie invariance algebra for each system and then find the kernel invariance algebra.

Thirdly, find all $G^{\sim}$-inequivalent Lie symmetry extensions of the kernel invariance algebra and all the $G^{\sim}$-inequivalent values of the arbitrary elements $\theta$. This last step gives us canonical forms for the Linear Schrödinger equations.

\subsection{Summary of the papers}

\section{Paper I: Algebraic method for group classification of (1+1)-dimensional linear Schrödinger equations with complex potentials}

In this paper we carry out the complete group classification of the class of linear $(1+1)$-dimensional Schrödinger equations with complex potentials,

$$
i \psi_{t}+\psi_{x x}+V(t, x) \psi=0
$$

where $\psi$ is the complex-valued unknown function of the variables $t \in \mathbb{R}$ and $x \in \mathbb{R}$, and the complex-valued smooth parameter-function $V$ of $(t, x)$ is interpreted as potential.

We find the equivalence groupoid $\mathcal{G}^{\sim}$, the equivalence group $G^{\sim}$ and the equivalence algebra $\mathfrak{g}^{\sim}$ of this class, which allows us to prove that the class is uniformly semi-normalized.

The next step is to study vector fields that generate the Lie point symmetries for a given equation $\mathcal{L}_{V}$ from this class with a given potential $V=V(t, x)$. The splitting with respect to the potential $V$ and its derivatives leads to a system of linear partial differential equations for the coefficients of the vector fields that are infinitesimal symmetries for each potential $V$. The integration of this system gives the kernel invariance algebra $\mathfrak{g}^{\cap}$.

We then turn to finding inequivalent Lie symmetry extensions of the kernel algebra $\mathfrak{g}^{\cap}$. To do this, we consider the linear span $\mathfrak{g}_{\langle\rangle}=\sum_{V} \mathfrak{g}_{V}$ when $V$ varies. $\mathfrak{g}_{\langle\rangle}$is a Lie algebra and can be represented as a semi-direct sum $\mathfrak{g}_{\langle\rangle}^{\text {ess }} \in \mathfrak{g}_{\langle\rangle}^{\text {lin }}$ of a subalgebra $\mathfrak{g}_{\langle\rangle}^{\text {ess }}$ and an abelian ideal $\mathfrak{g}_{\langle\rangle}^{\text {lin }}$. For each potential $V$, this splitting induces a similar representation for the maximal Lie invariance algebra $\mathfrak{g}_{V}$, which is $\mathfrak{g}_{V}=\mathfrak{g}_{V}^{\text {ess }} \in \mathfrak{g}_{V}^{\text {lin }}$, where $\mathfrak{g}_{V}^{\text {ess }}$ is a finite-dimensional Lie algebra and $\mathfrak{g}_{V}^{\text {lin }}$ is an infinite dimensional abelian ideal of $\mathfrak{g}_{V}$. We then establish the relation $\mathfrak{g}_{\langle\rangle}^{\text {ess }}=\pi_{*} \mathfrak{g}^{\sim}$ where $\pi$ is the projection onto the space with local coordinates $(x, u)$. We also prove that $\operatorname{dim} \mathfrak{g}_{V}^{\text {ess }} \leqslant 7$ for any $V$.

Finally, we introduce two invariant integers $k_{1}:=\operatorname{dim} \pi_{*}^{0} \mathfrak{g}_{V}^{\text {ess }} \in\{0,1,2,3\}$, where $\pi^{0}$ denotes the projection on the space of the variable $t$, and $k_{2}:=\operatorname{dim} \mathfrak{g}_{V}^{\text {ess }} \cap$ $\langle G(\chi), \sigma M, \rho I\rangle-2 \in\{0,2\}$. Using these integers, we complete the solution of the problem under study. These results are given in Theorem 21 of the paper. 


\section{Paper II: Group classification of multidimensional linear Schrödinger equations with algebraic method}

In this paper we consider the group classification problem for Schrödinger equations with complex potentials in dimension $(1+n)$ with $n \geqslant 2$,

$$
i \psi_{t}+\psi_{a a}+V(t, x) \psi=0
$$

where $\psi$ is the complex-valued unknown function of the variables $t \in \mathbb{R}$ and $x \in \mathbb{R}^{n}$, and the complex-valued smooth parameter-function $V$ of $(t, x)$ is interpreted as potential. The subscripts $t$ and $a$ denote differentiation with respect to $t$ and $x_{a}$, respectively, an the index $a$ runs from 1 to $n$. We use the summation convention, so $\psi_{a a}$ represents $\Delta \psi$.

We find the equivalence groupoid and the equivalence group of the given class in closed form and then show that the class is (uniformly) semi-normalized. This class admits the similar kernel invariance algebra $\mathfrak{g}^{\cap}$ as the corresponding class investigated in Paper I. We proceed to the calculation of the maximal Lie invariance algebra $\mathfrak{g}_{V}$ of the Schrödinger equation (2.4) for each fixed $V$. The algebra $\mathfrak{g}_{V}$ may by essentially higher-dimensional than the algebras obtained in Paper I for the case of $(1+1)$ variables. Moreover, the structure of $\mathfrak{g}_{V}$ may be much more complicated due to appearing rotations in higher-dimensional spaces.

We show that the span $\mathfrak{g}_{\langle\rangle}=\sum_{V} \mathfrak{g}_{V}$ is a Lie algebra and can be represented as the semi-direct sum $\mathfrak{g}_{\langle\rangle}^{\text {ess }} \in \mathfrak{g}_{\langle\rangle}^{\text {lin }}$ of a subalgebra $\mathfrak{g}_{\langle\rangle}^{\text {ess }}$ and an abelian ideal $\mathfrak{g}_{\langle\rangle}^{\text {lin }}$, just as in Paper I.

In order to find Lie symmetry extensions of the kernel invariance algebra $\mathfrak{g}^{\cap}$, we apply the algebraic method and reduce the group classification problem to the classification of certain low-dimensional subalgebras of the associated equivalence algebra. An important role in the consideration is played by estimates of the dimension of the essential Lie invariance algebra $\mathfrak{g}_{V}^{\text {ess }}$ and its various parts for any potential $V$. In particular,

$$
\operatorname{dim} \mathfrak{g}_{V}^{\mathrm{ess}} \leqslant \frac{n(n+3)}{2}+5
$$

The group classification of linear Schrödinger equations in the (1+2)-dimensional case is considered in more detail. For the exposition to be systematic, we the introduce the three $\pi G^{\sim}$-invariant integers

$$
\begin{aligned}
& k_{1}:=\operatorname{dim} \pi_{*}^{0} \mathfrak{g}_{V}^{\text {ess }} \in\{0,1,2,3\}, \\
& k_{2}:=\operatorname{dim} \pi^{J}\left(\mathfrak{g}_{V}^{\text {ess }} \cap\langle J, G(\chi), \sigma M, \rho I\rangle\right) \in\{0,1\}, \\
& k_{3}:=\operatorname{rank}\left\{\chi \mid \exists \sigma, \rho: G(\chi)+\sigma M+\rho I \in \mathfrak{g}_{V}^{\text {ess }}\right\} \in\{0,1,2\},
\end{aligned}
$$

where $\pi^{0}$ and $\pi^{J}$ denote the projections on the space of the variable $t$ and on the span $\langle J\rangle$, respectively. Using these integers, we obtain a complete classification of inequivalent Lie symmetry extensions for $(1+2)$-dimensional linear Schrödinger equations with complex-valued potentials. These results are given in Theorem 18 of the paper. 


\section{Bibliography}

[1] Bender C.M., Making sense of non-Hermitian Hamiltonians, Rep. Progr. Phys. 70 (2007), 947-1018.

[2] Bender C.M., Brody D.C. and Jones H.F., Complex extension of quantum mechanics, Phys. Rev. Lett. 89 (2002), 270401, 4 pp.

[3] Bihlo A., Dos Santos Cardoso-Bihlo E. and Popovych R.O., Complete group classification of a class of nonlinear wave equations, J. Math. Phys. 53 (2012), $123515,32 \mathrm{pp}$.

[4] Bluman G.W. and Kumei S., Symmetries and differential equations, SpringerVerlag, New York, 1989.

[5] Boyer C.P., The maximal 'kinematical' invariance group for an arbitrary potential, Helv. Phys. Acta 47 (1974), 589-605.

[6] Fernández F.M., Guardiola R., Ros J. and Znojil M., A family of complex potentials with real spectrum, J. Phys. A 32 (1999), 3105-3116.

[7] Fushchich W.I. and Moskaliuk S.S., On some exact solutions of the nonlinear Schrödinger equation in three spatial dimensions, Lett. Nuovo Cimento (2) 31 (1981), 571-576.

[8] Gagnon L. and Winternitz P., Symmetry classes of variable coefficient nonlinear Schrödinger equations, J. Phys. A 26 (1993), 7061-7076.

[9] Hydon P.E., Symmetry methods for differential equations. A beginner's guide, Cambridge University Press, Cambridge, 2000. 
[10] Ivanova N., Symmetry of nonlinear Schrödinger equations with harmonic oscillator type potential, in Symmetry in nonlinear mathematical physics, Part 1, 2 (Kyiv, 2001), vol. 2 of Pr. Inst. Mat. Nats. Akad. Nauk Ukr. Mat. Zastos., 43, Part 1, Natsīonal. Akad. Nauk Ukraïni, İnst. Mat., Kiev, pp. 149-150, 2002.

[11] Kingston J.G. and Sophocleous C., On point transformations of a generalised Burgers equation, Phys. Lett. A 155 (1991), 15-19.

[12] Kingston J.G. and Sophocleous C., On form-preserving point transformations of partial differential equations, J. Phys. A 31 (1998), 1597-1619.

[13] Kurujyibwami C., Equivalence groupoid for (1+2)-dimensional linear Schrödinger equations with complex potentials, J. Phys.: Conf. Ser. 621 (2015), 12008-12014.

[14] Lie S., Über die Integration durch bestimmte Integrale von einer Klasse linear partieller Differentialgleichung, Arch. for Math. 6 (1981), 328-368.

[15] Miller Jr. W., Symmetry and separation of variables, Addison-Wesley Publishing Co., Reading, Mass.-London-Amsterdam, 1977.

[16] Mostafazadeh A., A dynamical formulation of one-dimensional scattering theory and its applications in optics, Ann. Physics 341 (2014), 77-85.

[17] Niederer U., The maximal kinematical invariance group of the free Schrödinger equation, Helv. Phys. Acta 45 (1972), 802-810.

[18] Niederer U., The maximal kinematical invariance group of the harmonic oscillator, Helv. Phys. Acta 46 (1973), 191-200.

[19] Niederer U., The maximal kinematical invariance groups of Schrödinger equations with arbitrary potentials, Helv. Phys. Acta 47 (1974), 167-172.

[20] Oliveri F., Lie symmetries of differential equations: classical results and recent contributions, Symmetry 2 (2010), 658-706.

[21] Olver P.J., Applications of Lie groups to differential equations, SpringerVerlag, New York, 1993.

[22] Ovsiannikov L.V., Group analysis of differential equations, Academic Press, New York-London, 1982.

[23] Pommaret J.F., Systems of partial differential equations and Lie pseudogroups, Gordon \& Breach Science Publishers, New York, 1978.

[24] Popovych R.O., Kunzinger M. and Eshraghi H., Admissible transformations and normalized classes of nonlinear Schrödinger equations, Acta Appl. Math. 109 (2010), 315-359.

[25] Saunders D.J., The geometry of jet bundles, Cambridge University Press, Cambridge, 1989. 


\section{Part II}

\section{Research papers}





\section{Papers}

The articles associated with this thesis have been removed for copyright reasons. For more details about these see:

http://urn.kb.se/resolve?urn=urn:nbn:se:liu:diva-125136 